\title{
Cell-element simulations to optimize the performance of osmotic processes in porous membranes
}

\author{
Victor M. Calo ${ }^{\mathrm{a}, \mathrm{b}}$, Oleg Iliev ${ }^{\mathrm{c}, \mathrm{d}}$, Suzana P. Nunes ${ }^{\mathrm{e}}$, Galina Printsypar ${ }^{\mathrm{f}, *}$, Meixia Shi ${ }^{\mathrm{e}}$ \\ ${ }^{a}$ Curtin University, Applied Geology Department, Western Australian School of Mines, Faculty of Science and Engineering, \\ Perth, WA 6845, Australia \\ ${ }^{b}$ Mineral Resources, Commonwealth Scientific and Industrial Research Organization (CSIRO), Kensington, WA 6152, \\ Australia \\ ${ }^{c}$ Flows and Materials Simulation Department, Fraunhofer Institute for Industrial Mathematics, Kaiserslautern 67663, \\ Germany \\ ${ }^{d}$ Institute of Mathematics, BAS, Acad. G. Bonchev str., Bldg. 8, Sofia 1113, Bulgaria \\ ${ }^{e}$ Biological and Environmental Science and Engineering Division, King Abdullah University of Science and Technology, \\ Thuwal 23955-6900, Saudi Arabia \\ ${ }^{f}$ Mathematical Institute, University of Oxford, Oxford OX2 6GG, UK
}

\begin{abstract}
We present a new module of the software tool PoreChem for 3D simulations of osmotic processes at the cell-element scale. We consider the most general fully coupled model (see e.g., [1]) in 3D to evaluate the impact on the membrane performance of both internal and external concentration polarization, which occurs in a cell-element for different operational conditions. The model consists of the Navier-StokesBrinkman system to describe the free fluid flow and the flow within the membrane with selective and support layers, a convection-diffusion equation to describe the solute transport, and nonlinear interface conditions to fully couple these equations. First, we briefly describe the mathematical model and discuss the discretization of the continuous model, the iterative solution, and the software implementation. Then, we present the analytical and numerical validation of the simulation tool. Next, we perform and discuss numerical simulations for a case study. The case study concerns the design of a cell element for the forward osmosis experiments. Using the developed software tool we qualitatively and quantitatively investigate the performance of a cell element that we designed for laboratory experiments of forward osmosis, and discuss the differences between the numerical solutions obtained with the full 3D and reduced 2D models. Finally, we demonstrate how the software enables investigating membrane heterogeneities.
\end{abstract}

Keywords: Forward osmosis, mathematical modeling, cell element, porous membranes, heterogeneous and defective selective layer

\section{Introduction}

Forward osmosis (FO) and pressure retarded osmosis (PRO) are industrial processes that rely on the osmotic pressure difference across a semi-permeable membrane [2]. The osmotic pressure difference drives both processes, but unlike in FO, PRO occurs in the presence of a non-negligible hydraulic pressure. FO is used in, for example, hybrid systems for waste water, in agriculture, in food processes [3-5], while PRO is used in, e.g., the technology for renewable power generation $[6,7]$.

Concentration polarization significantly affects the efficiency of both processes. Concentration polarization is a concentration gradient which occurs next to the surface of the membrane or inside the support layer.

\footnotetext{
* Corresponding author

Email addresses: victor.calo@curtin.edu.au (Victor M. Calo), oleg.iliev@itwm.fhg.de (Oleg Iliev), suzana.nunes@kaust.edu.sa (Suzana P. Nunes), galina.printsypar@maths.ox.ac.uk (Galina Printsypar), meixia.shi@kaust.edu.sa (Meixia Shi)
} 
The main driving force in FO and PRO is the osmotic pressure which directly depends on the concentration difference across the separating membrane interface, the polarization phenomenon has a significant impact on the performance of these osmotic processes. There are two types of concentration polarization, internal (ICP), when the concentration gradient is found inside the support layer, and external (ECP), when the concentration gradient is found next to the membrane surface $[3,8]$.

The membrane performance is usually evaluated using a cross-flow cell element, which is a lab-scale filtration unit designed to measure an accurate performance while using a minimal membrane area. Laboratory experiments for osmotic processes provide measurements only at the output of a cell element, while the details of the processes within the cell element are usually not accessible. In particular, concentration polarization cannot be easily measured because it occurs within the cell element, thus, it is usually evaluated indirectly. Mathematical modeling followed by computer simulations is a popular approach in the membrane community, which assists in the design and interpretation of the laboratory experiments and also helps to better understand the osmotic processes. A mathematical model which is correctly formulated and accurately applied provides a detailed 3D picture of the processes, leading to their adequate characterization.

Several factors control the concentration polarization, namely the shape of a cell element in the laboratory or a membrane module in industrial applications, the flow control parameters, and membrane characteristics [9]. These factors can be accounted explicitly or implicitly while studying concentration polarization. Below we review the models introduced in the literature, and comment on their advantages and limitations.

\section{Modeling assumptions}

To numerically represent concentration polarization, most of the studies (see for example $[3,8,10-14]$ ) use directly or modify the ICP model initially proposed for PRO phenomena by Lee et al. [15] and then extended for FO by Loeb et al. in [16]. The model describes the transport through the membrane taking into account ICP and membrane orientation. The model analytically solves the governing equations within the membrane while assuming that the osmotic pressure depends linearly on the concentration, that is, it assumes as ideal solution. The linearity assumption, however, can be applied only in limited number of cases, since several studies showed that the osmotic pressure-concentration relation is approximately linear only for low concentration solutions. For high concentration solutions, which are most relevant for FO and PRO processes, a more complex approximation is needed [3, 17, 18].

The limitations of the linearity assumptions inspired extensions of this simple model. Tang et al. [19] proposed an iterative model to account for the internal concentration polarization in forward osmosis that circumvents the linearity assumption between the osmotic pressure and the concentration. This method uses an analytical solution of the governing equations for the flow and the transport inside the membrane coupled with a nonlinear osmotic pressure-concentration relation. Since the governing equations are stated and solved only for the membrane, the proposed approach accounts only for internal effects of the concentration, but not for external ones. Nevertheless, the external concentration polarization has a significant impact on performance (see e.g., [3]). Later, this analytical model was incorporated into an FO model for the whole cross-flow setup that accounts for fluid dynamics and solute transport [17]. The latter approach accounts for the ECP influence, but has two limitations that cannot be overcome. First, the analytical solution in the membrane assumes that the hydraulic pressure is negligible, and therefore only the solute transport is accounted for in the support layer, while fluid flow effects are explicitly neglected from the model. Hence, the model cannot be directly applied to PRO processes. Second, all parameters of the support layer are averaged over its thickness, therefore, complex heterogeneities cannot be included.

To simulate forward osmosis processes Sagiv et al. [1, 20] used a general model that does not rely on the linearity assumption and considers a resolved support layer of the membrane. This model generalizes the previously discussed approaches as it accounts for arbitrary osmotic pressure-concentration relations and has the potential to be further extended to model pressure retarded osmosis processes or to investigate membrane heterogeneities. Keeping in mind these advantages, we choose this mathematical model as a base model for our study. 


\section{Dimensionality of simulations}

Another aspect which deserves discussion in connection with existing numerical simulations of FO and PRO processes is their dimensionality. The interpretation and design of experimental and of some numerical studies concerned with FO and PRO processes usually assume that the geometry of the cell elements produces a homogeneous tangential flow. Based on this presumption, the cell element geometry is assumed not to affect the membrane performance, i.e., any 3D effects are ignored. Under this homogeneity assumption, experimental studies rarely present or discuss the geometry of the cell elements that were used in the experiments (a notable exception is the experimental work [21]). Thus, it is difficult to evaluate the reliability of the parameters extracted from these experiments. While this presumption may be is valid in many cases, accepting it as always valid is dangerous.

A part of this paper describes our efforts to design a cell element for which the homogeneity assumption is not severely violated. But some cell elements might introduce a significant error if their 3D shape is neglected. Sagiv et al. [22] analyzed effects of the different factors on water permeation of the forward osmosis, and in particular, they found that improving the cell element design is beneficial for the forward osmosis performance for the parameters and regimes they investigated. We should note, however, that although in their study the ICP within the support layer did not play essential role, we found [23] that for other regimes ICP can have a significant impact.

All the numerical studies that we discussed above are carried out either for 1D or 2D cases except [13, 14]. There, the authors investigate two different cell elements and observe significant influence of their shapes on the flux distribution and external concentration polarization. However, the mathematical model used in $[13,14]$, as discussed above, has limitations.

\section{Goals and organization of the paper}

The goal of the paper is twofold: (I) to present a new module of the software tool PoreChem, which we develop for $3 \mathrm{D}$ simulation of osmotic processes at cell-element scale, including its validation; (II) to present and to discuss results of a case study, that uses this software tool to design of a cell element for the laboratory experiments and to support reliable interpretation of the measurements. In addition, we illustrate the capabilities of the software tool to investigate the effects of membrane heterogeneities (using current technologies, heterogeneities in membranes manufactured for laboratory testing and especially for industrial purposes are unavoidable).

To the best of our knowledge, no other paper discusses numerical simulation of osmotic processes in full $3 \mathrm{D}$ cell element geometry using a general mathematical model as the one from [1]. The presented case study on the cell element performance, as well as the study of the influence of the membrane heterogeneity and defects, illustrate the capability of the developed software platform, namely PoreChem [24, 25], and extend our understanding of the osmotic processes and the factors influencing their performance.

The rest of the paper is organized as follows. In the next section we present the problem setup and the mathematical model in detail. The third section describes the analytical and numerical validation of the algorithm and the implementation. Those who are not interested in such discussion can skip this section without loss of understanding of the further sections. A case study is presented in the fourth section. More precisely, we show how the shape of the cell element influences the concentration polarization, and thus, the membrane performance in forward osmosis processes. We also discuss the impact of the dimensionality of the used mathematical model. In the appendix we present some numerical tests which demonstrate how the developed 3D mathematical model can be exploited to study the impact of membrane heterogeneity and defects on its performance.

\section{Mathematical model}

Let us first introduce some notation. The computational domain (see Figure 1) is denoted by $\Omega$ and consists of two non-overlapping domains, namely the fluid domain $\Omega_{f}$ and the porous domain $\Omega_{p}$, so that $\Omega=\Omega_{f} \cup \Omega_{p}$. The boundary $\partial \Omega$ is composed of three different regions. We denote the inlet and outlet boundaries by $\Gamma^{\text {in }}$ and $\Gamma^{\text {out }}$, respectively, and represent the solid walls by $\Gamma^{s}$, where $\partial \Omega=\Gamma^{s} \cup \Gamma^{\text {in }} \cup \Gamma^{\text {out }}$. 


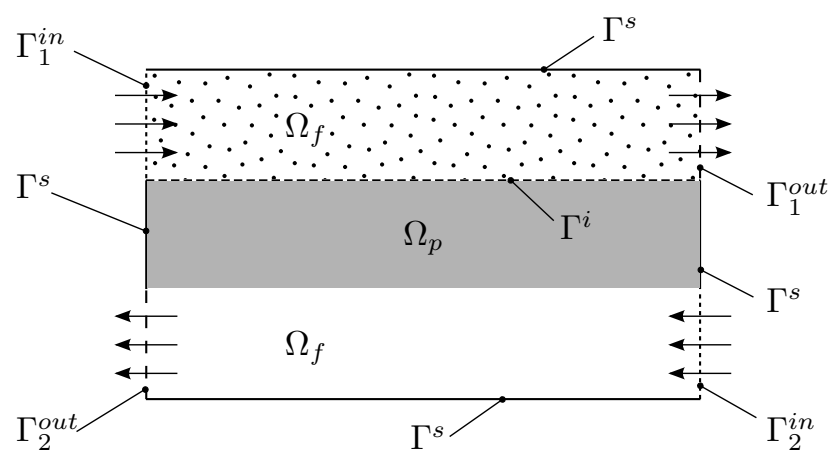

Figure 1: Counter-current cross-flow setup in AL-DS mode.

Thin film composite membranes are usually used for forward osmosis. These thin membranes consist at least of two layers, namely a selective or active layer and a support layer. The main functionality of the thin selective layer is to reject the solute, while the support layer provides mechanical support for the selective layer. Both layers influence the performance of the membrane. FO can use two membrane orientations, because the membrane is asymmetric. The modes, when the selective layer faces the draw and feed solutions, are commonly referred as AL-DS and AL-FS, respectively. Using one or other preferential orientation can help for instance to minimize fouling in FO systems operating with feed or draw solutions having potential foulants such as wastewater. We do not consider fouling in this work, we only consider the effects of concentration polarization on the performance of the membrane system.

We model an FO cross-flow setup (see a 2D sketch in Figure 1) with the selective layer of the membrane facing the draw solution (AL-DS mode). There are two channels, one with a draw solution (upper) and the other one with a feed solution (lower), separated by a membrane. The membrane consists of two layers, namely the support layer $\Omega_{p}$ and the selective layer $\Gamma^{i}$, which we model as a zero-thickness interface between the support layer and the draw channel. The fluid flows tangentially to the membrane along the channels in co- or counter-current directions, while close to the selective layer the permeate velocity is normal to the membrane surface.

We assume that the fluid is Newtonian, the flow is laminar and incompressible, and the process is isothermal. For this case there exist different models for simulating a free fluid flow coupled with a flow in porous media. Popular approaches use Stokes and Darcy flow models with interface coupling conditions (see e.g. [26] and references therein). Another approach is to use the Navier-Stokes-Brinkman model for the fluid flow through a filter element [27]. We use the latter approach. The steady Navier-Stokes-Brinkman equations read

$$
\begin{aligned}
-\nabla \cdot\left(\mu_{e f f} \nabla \mathbf{u}\right)+(\rho \mathbf{u} \cdot \nabla) \mathbf{u}+\mu \mathbf{K}^{-1} \mathbf{u} & =-\nabla p, & & \mathbf{x} \in \Omega ; \\
\nabla \cdot \mathbf{u} & =0, & & \mathbf{x} \in \Omega .
\end{aligned}
$$

Here $\mathbf{u}$ and $p$ denote the fluid velocity vector in $[\mathrm{m} / \mathrm{s}]$ and the fluid pressure in $[P a]$, respectively. Moreover, $\mu$ and $\mu_{e f f}$ are the fluid dynamic viscosity and the effective viscosity measured in $[\mathrm{Pas}]$. $\mathbf{K}$ is the intrinsic permeability of the porous medium in $\Omega_{p}$ and $\mathbf{K}^{-1}=0$ in $\Omega_{f}$.

A typical set of boundary conditions reads as follows

$$
\begin{aligned}
& \mathbf{u}(\mathbf{x})=\mathbf{u}^{i n}(\mathbf{x}), \quad \mathbf{x} \in \Gamma^{i n} ; \\
& \boldsymbol{\sigma} \cdot \mathbf{n}=0, \quad \mathbf{x} \in \Gamma^{\text {out }} ; \\
& \mathbf{u}(\mathbf{x})=\mathbf{0}, \quad \mathbf{x} \in \Gamma^{s} ;
\end{aligned}
$$

where $\boldsymbol{\sigma}=-p \mathbf{I}+\mu_{\text {eff }}\left(\nabla \mathbf{u}+(\nabla \mathbf{u})^{T}\right)$ is the stress tensor for incompressible viscous fluids, $\mathbf{n}$ is the outward unit normal to $\Gamma^{\text {out }}$. More details can be found, for example, in [27, 28].

The solute transport is modeled using the following convection-diffusion equation

$$
-\nabla \cdot(D \nabla c)+\nabla \cdot(\mathbf{u} c)=0, \quad \mathbf{x} \in \Omega .
$$


We denote the solute concentration by $c$ which is measured in $[M]=[\mathrm{mol} / L]$. The diffusion coefficient $D$ is measured in $\left[\mathrm{m}^{2} / \mathrm{s}\right]$ and can take different scalar values in $\Omega_{f}$ and in $\Omega_{p}$. In $\Omega_{f}, D$ is equal to the molecular diffusion of the solute in the liquid, while in $\Omega_{p}$ it is the effective diffusivity of the solute in porous media. The solute transport equation is supplemented with the following boundary conditions

$$
\begin{aligned}
c(\mathbf{x}) & =c^{i n}(\mathbf{x}), & & \mathbf{x} \in \Gamma^{\text {in }} ; \\
-D \nabla c \cdot \mathbf{n} & =0, & & \mathbf{x} \in \Gamma^{\text {out }} \cup \Gamma^{s} .
\end{aligned}
$$

We remark that equation (8) along with no-slip boundary condition (5) yields no-flux boundary condition on $\Gamma^{s}$, while equation (8) on $\Gamma^{\text {out }}$ yields only zero diffusive flux. The latter practically means that the concentration does not change at the outlet in the normal direction and the solute escapes the domain with the fluid convection.

The processes in the selective layer are modeled as interface conditions between the free fluid region and the support layer. We denote the interface as $\Gamma^{i}$ and introduce an operator $[f]_{\Gamma^{i}}$ which indicates a jump of the function $f$ across the interface $\Gamma^{i}$

$$
[f]_{\Gamma^{i}}=\lim _{\mathbf{x} \rightarrow \Gamma^{i}+0} f(\mathbf{x})-\lim _{\mathbf{x} \rightarrow \Gamma^{i}-0} f(\mathbf{x}) .
$$

Then, to model the selective layer we impose the following interfacial conditions, which restrict the solute and water fluxes through the membrane, along with the conditions for the continuity of fluxes

$$
\begin{aligned}
\mathbf{u}(\mathbf{x}) \cdot \mathbf{n} & =-\frac{A}{\mu s}\left([p]_{\Gamma^{i}}-\phi[\pi]_{\Gamma^{i}}\right), & {[\mathbf{u}(\mathbf{x}) \cdot \mathbf{n}]_{\Gamma^{i}}=0, } & \mathbf{x} \in \Gamma^{i} \\
\mathbf{J}_{s}(\mathbf{x}) \cdot \mathbf{n} & =-B[c]_{\Gamma^{i}}, & {\left[\mathbf{J}_{s}(\mathbf{x}) \cdot \mathbf{n}\right]_{\Gamma^{i}}=0, } & \mathbf{x} \in \Gamma^{i}
\end{aligned}
$$

where $\mathbf{J}_{s}=-D \nabla c+\mathbf{u} c$ is the solute flux in $[M \mathrm{~m} / \mathrm{s}] ; A\left(\left[\mathrm{~m}^{2}\right]\right)$ and $B([\mathrm{~m} / \mathrm{s}])$ are the water and solute permeability of the selective layer, respectively; $s$ is the thickness of the selective layer in $[m] ; \phi([-])$ is the reflection coefficient; $\pi$ is the osmotic pressure in $[K P a]$, which depends on the concentration.

Here, we note that the conservation of mass, i.e., the continuity of the solute flux $\mathbf{J}_{s}$, is preserved up to a discretization error thanks to the interfacial conditions (11) and a locally conservative finite volume scheme (see discussion below). At the same time, the concentration profile is discontinuous across the selective layer due to the solute permeability $B$ of the selective layer.

The mathematical model is implemented within PoreChem [24, 25]. The 3D computational geometry is discretized using an irregular rectangular grid. The spatial discretization uses a cell-centered finite volume method for the flow and solute transport models. To find the solution to Navier-Stokes-Brinkman system of equations time is introduced as an iteration counter and a Chorin-type algorithm iteratively imposes the incompressibility constraint. The flow and mass transport models are fully coupled via interfacial conditions (10) and (11) using an iterative procedure. The iterative procedure is performed until a prescribed accuracy $\epsilon$ is reached. Stopping criteria is the following

$$
\max \left(\left\{\frac{\left\|u_{i}^{n}-u_{i}^{n-1}\right\|}{\left\|u_{i}^{n}\right\|}\right\}_{i=x, y, z}, \frac{\left\|p^{n}-p^{n-1}\right\|}{\left\|p^{n}\right\|}, \frac{\left\|c^{n}-c^{n-1}\right\|}{\left\|c^{n}\right\|}\right)<\epsilon ;
$$

where $\|\cdot\|$ is the $l^{2}$-norm, the superscripts denote the time-step number. More details about the algorithm used to solve the discretized system of equations can be found in [25, 27, 29]. Specifically for this problem, we ensure the convergence of the iterative solver as follows. First, we find a steady-state solution for the coupled system with impermeable interfacial conditions (10) and (11), which means that we set $A$ and $B$ equal to zero. Then, we use this solution as an initial guess for the velocity, pressure, and concentration fields in the iterative solution of the coupled system with permeable interfacial conditions.

\section{Numerical validation}

Unlike mathematical validation, experimental validation of the model considered here and of the other simplified models discussed in the introduction is widely available in the literature, e.g., [1, 10, 17, 20]. To 
fill the gap, in this section we validate the model using available mathematical tests. First, we analyze the effect on the flow simulations of the chosen approximation of the selective layer, which physically has a finite thickness, by a zero-thickness interface conditions. Then, we investigate the accuracy of our flow solver by modeling a channel with a permeable wall for which an analytical solution is available.

\subsection{Selective layer model: thin dense layer versus interfacial conditions}

To validate the approximation of the thin dense selective layer as a zero-thickness interface condition, we perform a numerical experiment. We set up a co-current cross-flow channels with parameters close to those relevant for experimental setups when possible. A dense porous layer separates the flow channels. Its thickness is $50 \mu \mathrm{m}$, which is of the same order as that of conventional FO and PRO membranes. Here we only consider the fluid flow without the solute transport, thus we disregard the osmotic effects on the system. To generate a water flux through the porous layer using only hydraulic pressure, we set higher water permeability than the real selective layers usually have and use significantly larger flow rate in the upper channel. Then, we compare two cases: a resolved thickness of the dense layer, which is modeled as a 3D body, and the zero-thickness interface, which is two-dimensional, to represent the dense layer. In the first case, the Brinkman term in (1) restricts the water flow and models the porous layer, no interfacial conditions are used here. In the second case, the interfacial condition (10) is used to model the effect of the selective layer on the flow. In Table 1, we present the parameters used in the flow simulations. In the case of the 3D dense layer, the thickness specified in Table 1 is its actual thickness, while in the case of the interface, the thickness is an input parameter used in equation (10).

\begin{tabular}{|l|c|c|}
\hline Density $\rho$ & {$\left[\mathrm{kg} / \mathrm{m}^{3}\right]$} & 869 \\
Viscosity $\mu$ & {$[\mathrm{Pas}]$} & 0.0124 \\
Flow rate in the upper channel & {$[\mathrm{L} / \mathrm{min}]$} & 0.1 \\
Flow rate in the lower channel & {$\left[\mathrm{L} / \mathrm{min}^{-}\right]$} & $1 \times 10^{-5}$ \\
Pressure at the upper outlet & {$[\mathrm{KPa}]$} & 1 \\
Pressure at the lower outlet & {$[\mathrm{KPa}]$} & 0 \\
Membrane permeability K & {$\left[\mathrm{m}^{2}\right]$} & $1 \times 10^{-13}$ \\
Membrane thickness $s$ & {$[\mathrm{~mm}]$} & 0.05 \\
Computational domain & {$[\mathrm{mm}]$} & $5 \times 1 \times 1$ \\
Discretization step & {$[\mathrm{mm}]$} & 0.05 \\
\hline
\end{tabular}

Table 1: Input parameters for numerical experiment with the dense layer and the interface.
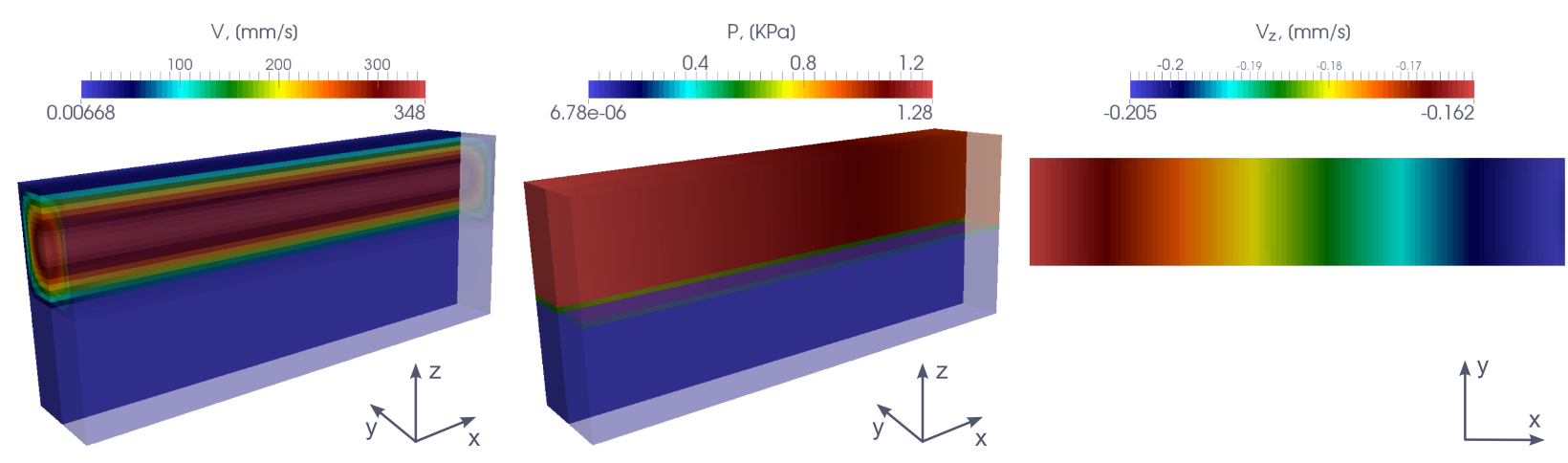

Figure 2: Co-current cross-flow setup with thin dense layer: distributions of the velocity magnitude, the pressure, and the vertical component of the velocity in the dense layer (from the left to the right).

In Figure 2, we show simulation results for the grid-resolved dense layer. The velocity, the pressure, and the vertical component of the velocity in the dense layer are represented from left to right, respectively. In Figure 3 (on the left), we compare the vertical component of the velocity through the dense layer and 

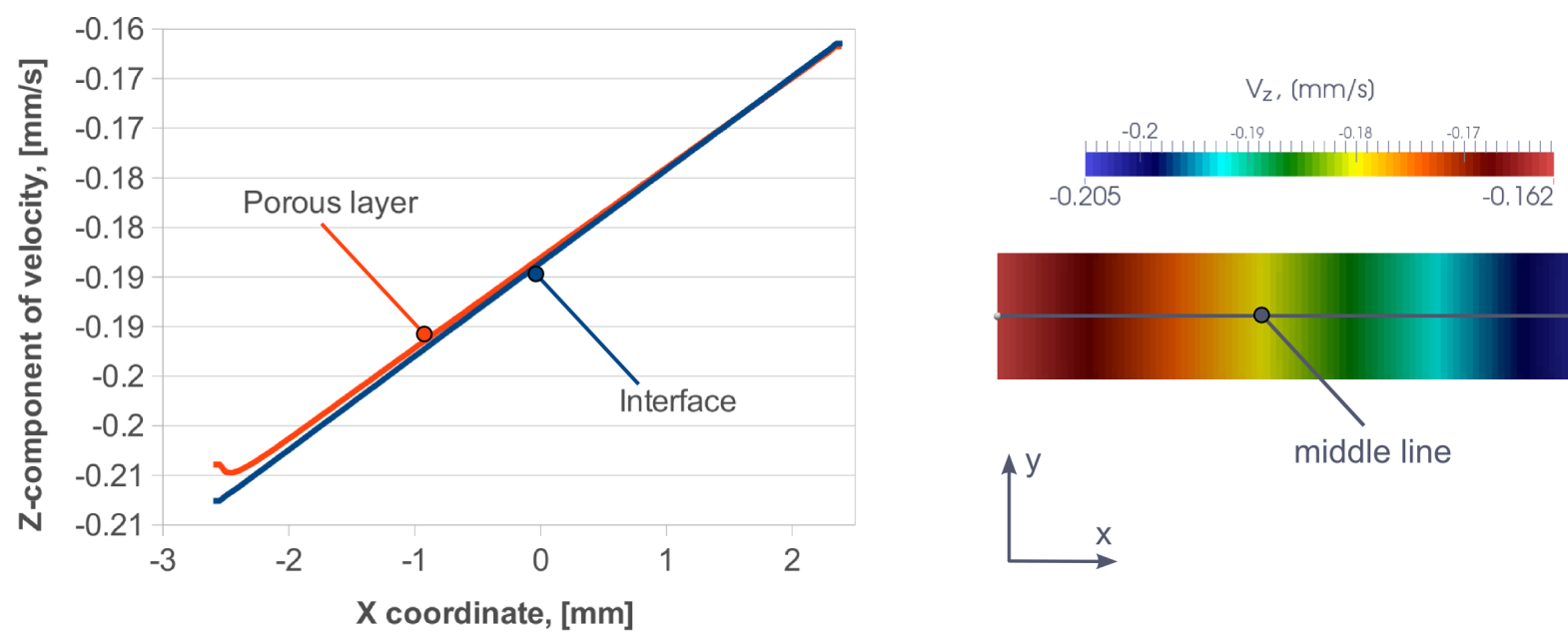

Figure 3: Distribution of the vertical velocity plotted over the middle line of the dense layer and the interface (on the left). On the right, the middle line is shown in the cross-section of the dense layer or the interface.

\begin{tabular}{|l|c|c|c|}
\hline & & Dense layer & Interface \\
\hline \multirow{2}{*}{ Pressure $p,[K P a]$} & $\min$ & $7.85 \times 10^{-6}$ & $6.78 \times 10^{-6}$ \\
& $\max$ & 1.29 & 1.28 \\
\hline \multirow{2}{*}{ Velocity magnitude $|\mathbf{u}|,[\mathrm{mm} / \mathrm{s}]$} & $\min$ & $6.63 \times 10^{-3}$ & $6.68 \times 10^{-3}$ \\
& $\max$ & 347 & 348 \\
\hline
\end{tabular}

Table 2: Results from the numerical experiments with the dense layer and the interface.

through the interface plotted over the middle line as shown in Figure 3 (on the right). In Table 2, we compare the minimum and maximum values of the pressure and the velocity obtained in both experiments. We observe good agreement between these two approaches (selective layer as a 3D body and as a 2D surface). We conclude that the thin selective layer is well represented as a zero-thickness interface for the chosen parameters, which are relevant for the applications of interest, namely forward osmosis and pressure retarded osmosis.

\subsection{Analytical and numerical solutions for a membrane in a cross-flow setup}

A 2D analytical solution is available for a channel with a membrane at the bottom [30]. Here, we use the analytical solution to evaluate the numerical one.

We setup the numerical experiment as shown in Figure 4. We have a cross-flow channel with a permeable bottom wall and model the membrane as a zero-thickness interface. The flow inlet is on the left, two flow outlets are positioned on the right and at the bottom. The 3D model is reduced to two dimensions by imposing periodic boundary conditions in the $y$ direction. Table 3 lists the parameters that are used in the numerical experiment.

The analytical solution assumes that the transverse flow velocity at any point is much smaller than the average longitudinal flow velocity, resulting in parabolic flow along the channel. To satisfy this assumption in the numerical experiment, our membrane is chosen to have very low permeability, which is also the case for forward osmosis membranes. Moreover, the analytical solution requires the pressure drop along the channel to be much smaller than that across the membrane in order to ensure a homogeneous permeation of the water through the membrane. In other words, we assume that the fluid velocity through the membrane has only a vertical component and is constant. See [30] for more details on the analytical solution. In general, the fluid velocity through the membrane is not always constant, for example see previous numerical experiment (Figure 3). To satisfy the second assumption, in the numerical experiment we choose the fluid pressure at the outlet of the channel itself to be much higher than at the outlet under the membrane (Table 3 ). 


\begin{tabular}{|l|c|c|l|c|c|}
\hline Density $\rho$ & {$\left[\mathrm{kg} / \mathrm{m}^{3}\right]$} & 1003 & Pressure at the upper outlet & {$[\mathrm{KPa}]$} & 5 \\
Viscosity $\mu$ & {$[\mathrm{Pas}]$} & 0.00089 & Pressure at the lower outlet & {$[\mathrm{KPa}]$} & 0 \\
Membrane thickness & {$[\mathrm{mm}]$} & 0.24 & Top channel size & {$[\mathrm{mm}]$} & $5 \times 0.3 \times 0.1$ \\
Membrane permeability K & {$\left[\mathrm{m}^{2}\right]$} & $4 \times 10^{-14}$ & Bottom channel size & {$[\mathrm{mm}]$} & $5 \times 0.3 \times 0.1$ \\
Flow rate & {$[\mathrm{L} / \mathrm{min}]$} & $3 \times 10^{-5}$ & Discretization step & {$[\mathrm{mm}]$} & 0.01 \\
\hline
\end{tabular}

Table 3: Input parameters for numerical experiment in $2 \mathrm{D}$ channel with constant outflow through the membrane.

\section{$V_{,}(\mathrm{mm} / \mathrm{s})$}

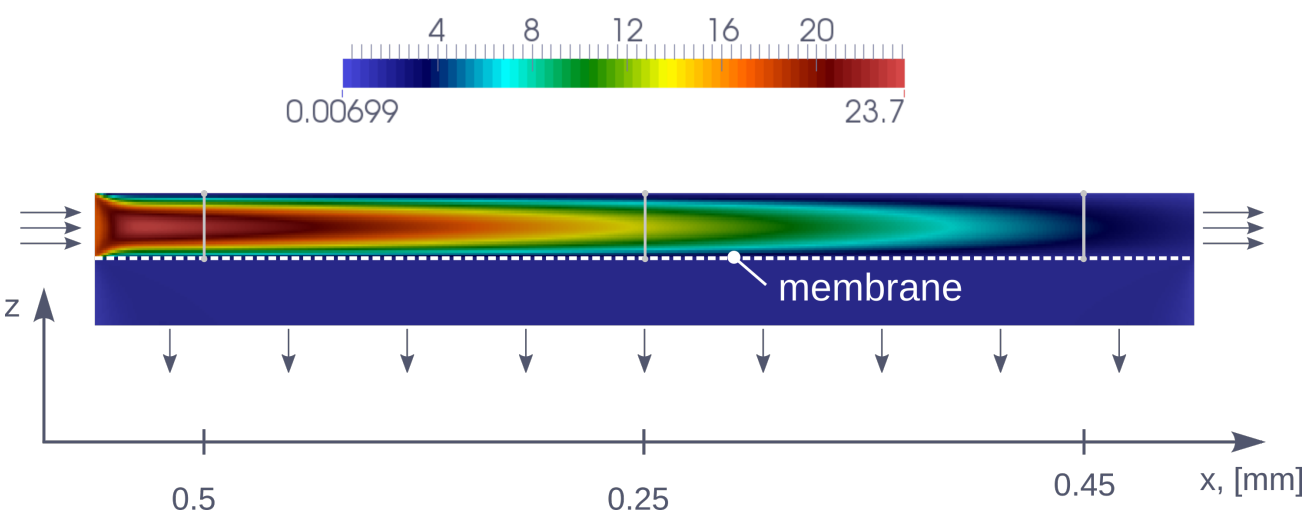

Figure 4: Velocity distribution obtained from the numerical experiment with some annotations.
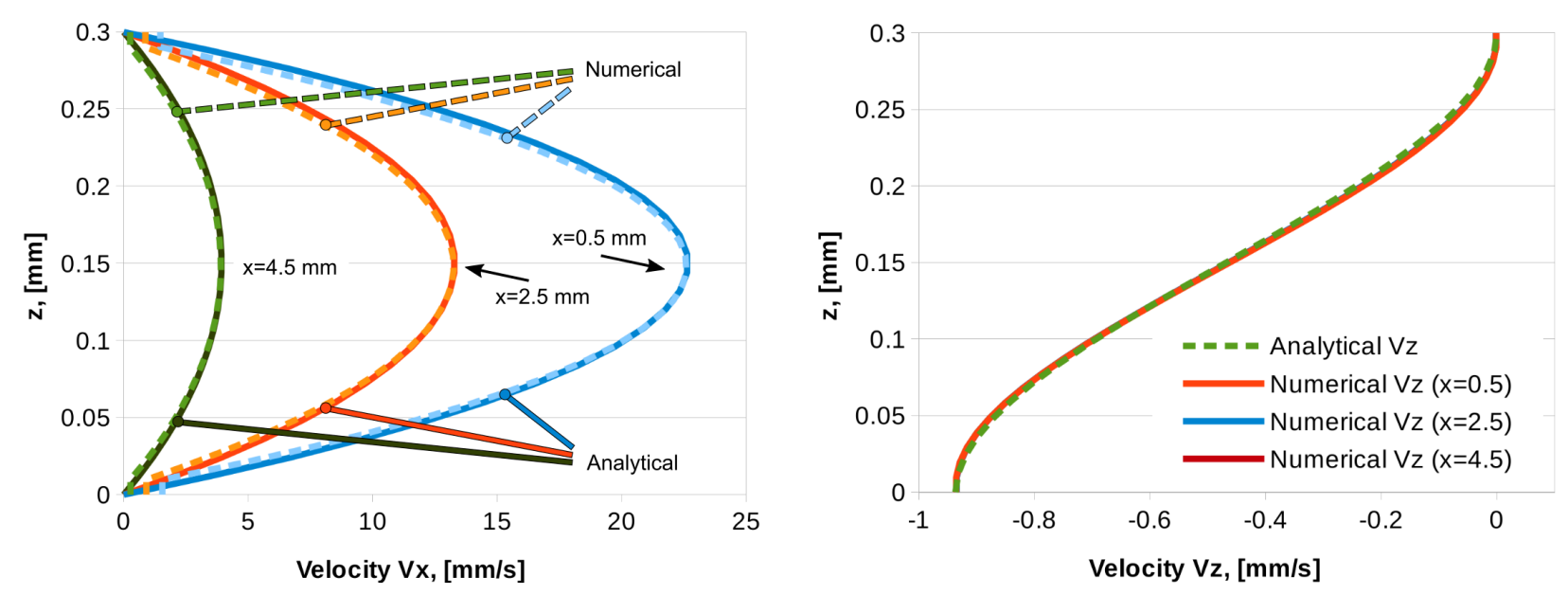

Figure 5: Comparison of analytical and numerical values of the horizontal and vertical velocity values.

Figure 4 shows the distribution of the fluid velocity in $x z$-plane obtained in the numerical simulation. To allow the flow to develop fully and minimize artifacts due to inlet and outlet boundary conditions, we remove from the comparison some distance in the $x$ direction from these boundaries. We plot the analytical and numerical velocity distributions at coordinate $x$ equal to $0.5,2.5$, and $4.5 \mathrm{~mm}$. Figure 5 shows these results. We observe a good agreement between the analytical and numerical velocity distributions, which indicates that the finite volume discretization delivers a reliable approximation to the mathematical model problem. 


\section{Cell-element numerical experiment}

We use the simulation tool described above to support the design of a cell element for forward osmosis. The goal is to design the cell element with low or negligible impact of the $3 \mathrm{D}$ effects on the membrane performance. The simulation results obtained only with the final design are presented here.

Performing forward osmosis experiments and postprocessing their results, most of the researchers usually assume that the flow is homogeneous and tangential to the membrane. In other words, the conditions of the experiment are assumed to be ideal, that is, 3D effects are neglected, and the forward osmosis is modeled as a two-dimensional process. If these assumptions are satisfied, the 2D study of the concentration polarization is easy, and the obtained values of the water and solute fluxes are reliable. In this section we focus on a numerical investigation of these assumptions.
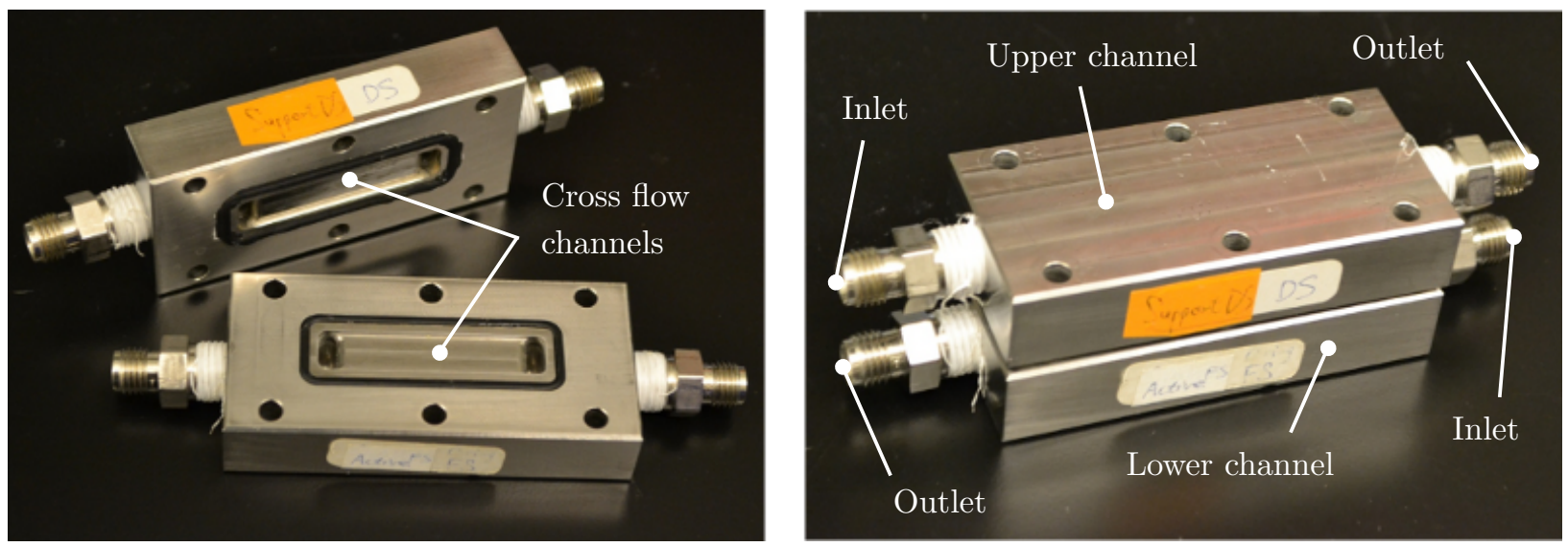

Figure 6: Experimental cell geometry: disassembled on the left and assembled on the right.
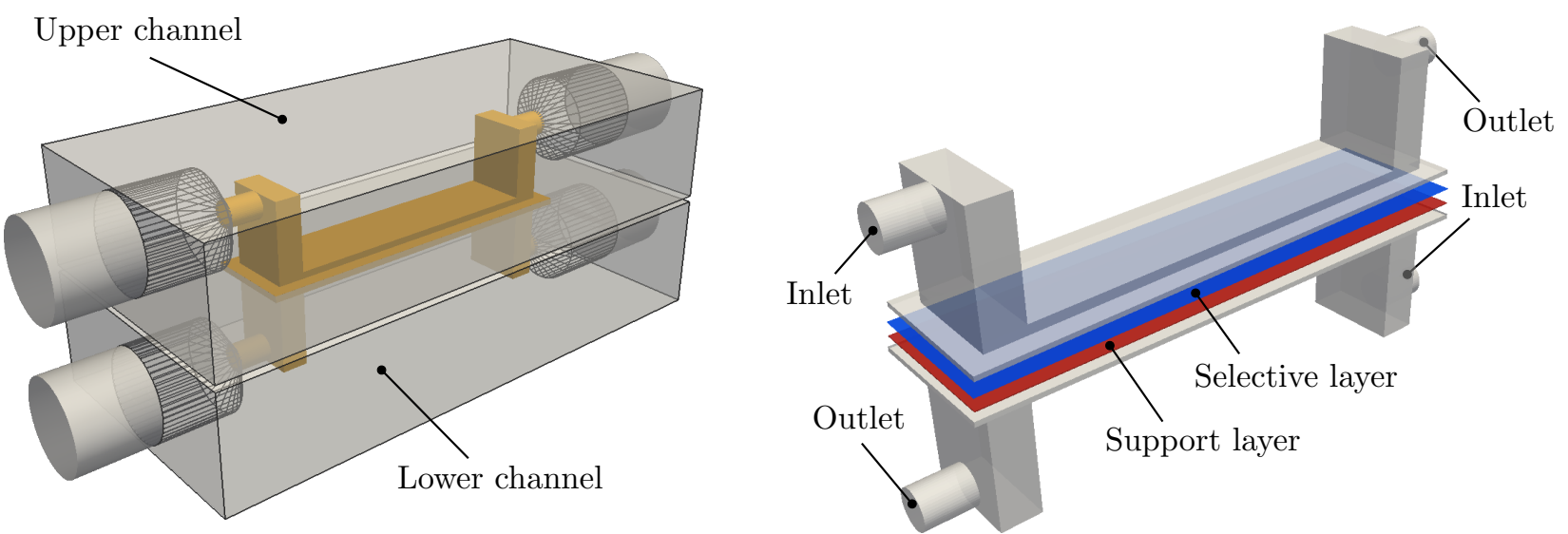

Figure 7: On the left, a digital prototype of the experimental cell element shown on the right of Figure 6 is presented. The fluid region that is considered for the simulations is marked in yellow. On the right, the magnified fluid region is shown with the inlets, outlets, the selective layer marked in blue, and the support layer marked in red.

\section{1. $3 D$ forward osmosis simulations}

First, we qualitatively investigate the influence of the 3D shape of the cell element by studying the distributions of the velocity and the concentration in the whole cell element and the distributions of the water and solute fluxes through the selective layer. We simulate numerically a forward osmosis experiment 


\begin{tabular}{|l|c|c|}
\hline Density $\rho$ & {$\left[\mathrm{kg} / \mathrm{m}^{3}\right]$} & 988.2071 \\
Viscosity $\mu$ & {$[\mathrm{Pas}]$} & 0.001002 \\
Flow rate in the draw channel & {$[\mathrm{L} / \mathrm{min}]$} & 0.1 \\
Flow rate in the feed channel & {$[\mathrm{L} / \mathrm{min}]$} & 0.1 \\
Concentration in the draw channel & {$[\mathrm{M}]$} & 2 \\
Concentration in the feed channel & {$[\mathrm{M}]$} & 0 \\
Diffusion coefficient $D$ in $\Omega_{f}$ & {$\left[\mathrm{~mm}^{2} / \mathrm{s}\right]$} & 0.001 \\
Diffusion coefficient $D$ in $\Omega_{p}$ & {$\left[\mathrm{~mm}^{2} / \mathrm{s}\right]$} & 0.00034 \\
Permeability of the support layer K & {$\left[\mathrm{m}^{2}\right]$} & $2.11 \times 10^{-17}$ \\
Thickness of the support layer & {$\left[\mathrm{mm}^{2}\right.$} & 0.12 \\
Water permeability of the selective layer $A$ & {$\left[\mathrm{~mm} \mathrm{~m}^{2}\right]$} & $2.0 \times 10^{-17}$ \\
Solute permeability of the selective layer $B$ & {$[\mathrm{~mm} / \mathrm{s}]$} & $1.3 \times 10^{-5}$ \\
Thickness of the selective layer $s$ & {$[\mathrm{~nm}]$} & 50 \\
Reflection coefficient $\phi$ & {$[-]$} & 1 \\
Osmotic pressure $\pi[31]$ & {$[\mathrm{KPa}]$} & $379.8 \mathrm{c}^{2}+4269 \mathrm{c}$ \\
Bounding box of the cell element & {$[\mathrm{mm}]$} & $14 \times 60 \times 26$ \\
\hline
\end{tabular}

Table 4: Input parameters for the numerical experiment for the cell element.

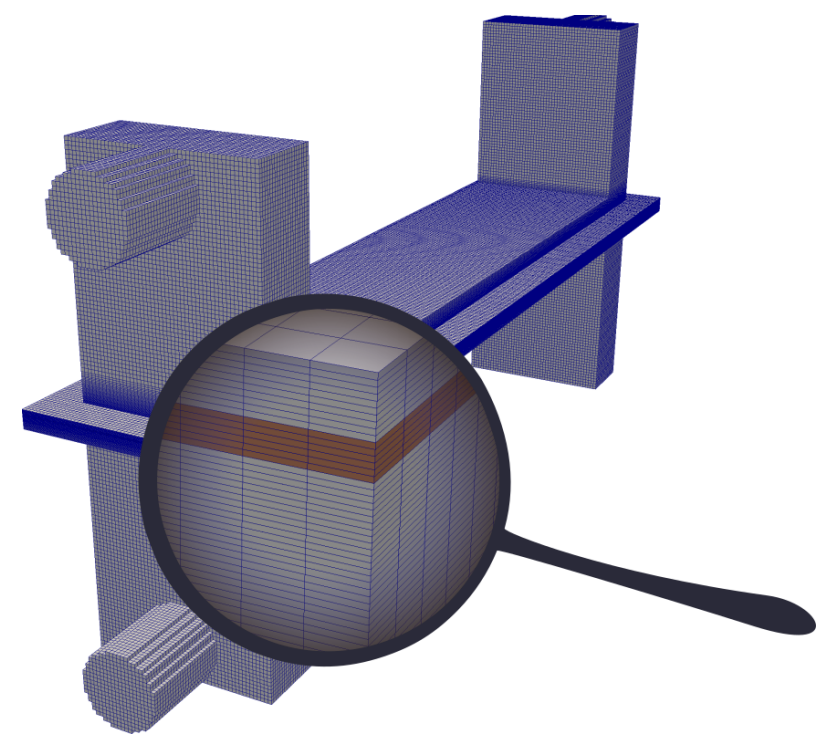

Figure 8: Irregular mesh for 3D simulations.

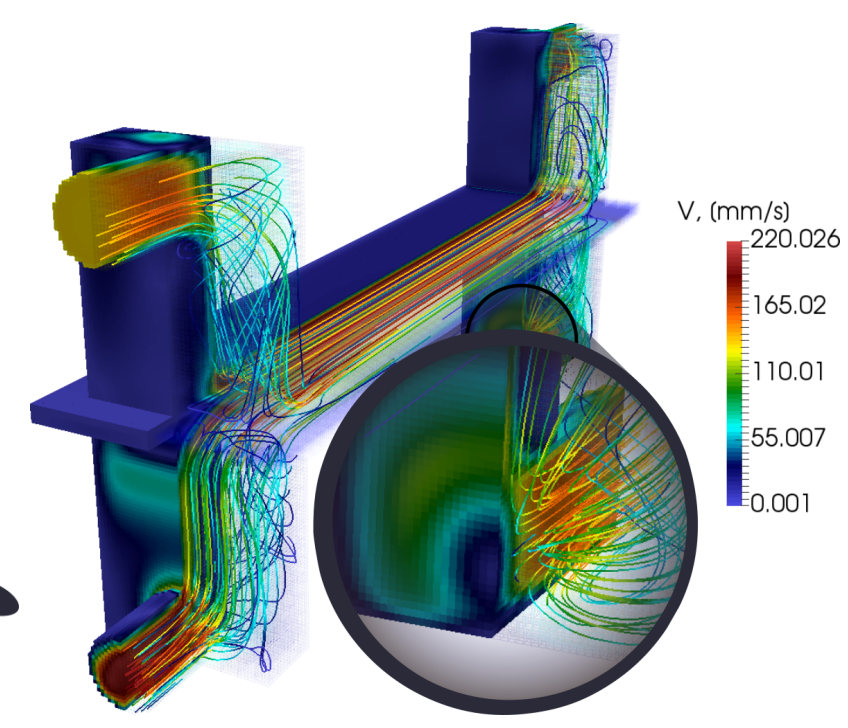

Figure 9: Velocity distribution in the cell element.

with the selective layer of the membrane facing the draw solution (i.e., an FO experiment in the AL-DS mode).

Figure 6 shows a photo of the real cell element used in the laboratory experiments. The left of Figure 6 shows two halves of the disassembled cell with the cross-flow channels. In an experiment the halves of the cell are assembled as shown on the right of Figure 6 with the membrane between them. For the countercurrent cross flow, the feed and draw solutions are pumped into the cell through the corresponding inlets, and come out of the outlets as the right of Figure 6 shows. To simulate this cell element, we generate a digital prototype of the geometry respecting the exact dimensions. Figure 7 shows on the left a digital prototype of the cell element with the fluid region that is used for the simulations marked in yellow. Figure 7 also details the fluid region with the red domain corresponding to the support layer and the blue interface on top of the support layer representing the selective layer.

The positions of the inlets and the outlets for the counter-current cross-flow setup are also shown on the 
right of Figure 7. We select the operational parameters to perform the simulations close to realistic values used in the experiments (see Table 4). To resolve the membrane keeping the number of unknowns under control, we use an irregular grid for the discretization (see Figure 8). The error tolerance $\epsilon=10^{-6}$ is used in the stopping criteria (12). The simulations take about $27 \mathrm{~h}$ to finalize.

Figure 9 shows the distributions of the water velocity in the cell element. Due to the shape of the cell element, we observe fast and tangential fluid flow close to the membrane surface only in the central part of the cell element. In the region below the inlet and the outlet, where the flow changes direction, the flow streamlines stop behaving tangentially. In the region close to the cell walls, we observe that the flow is slow.
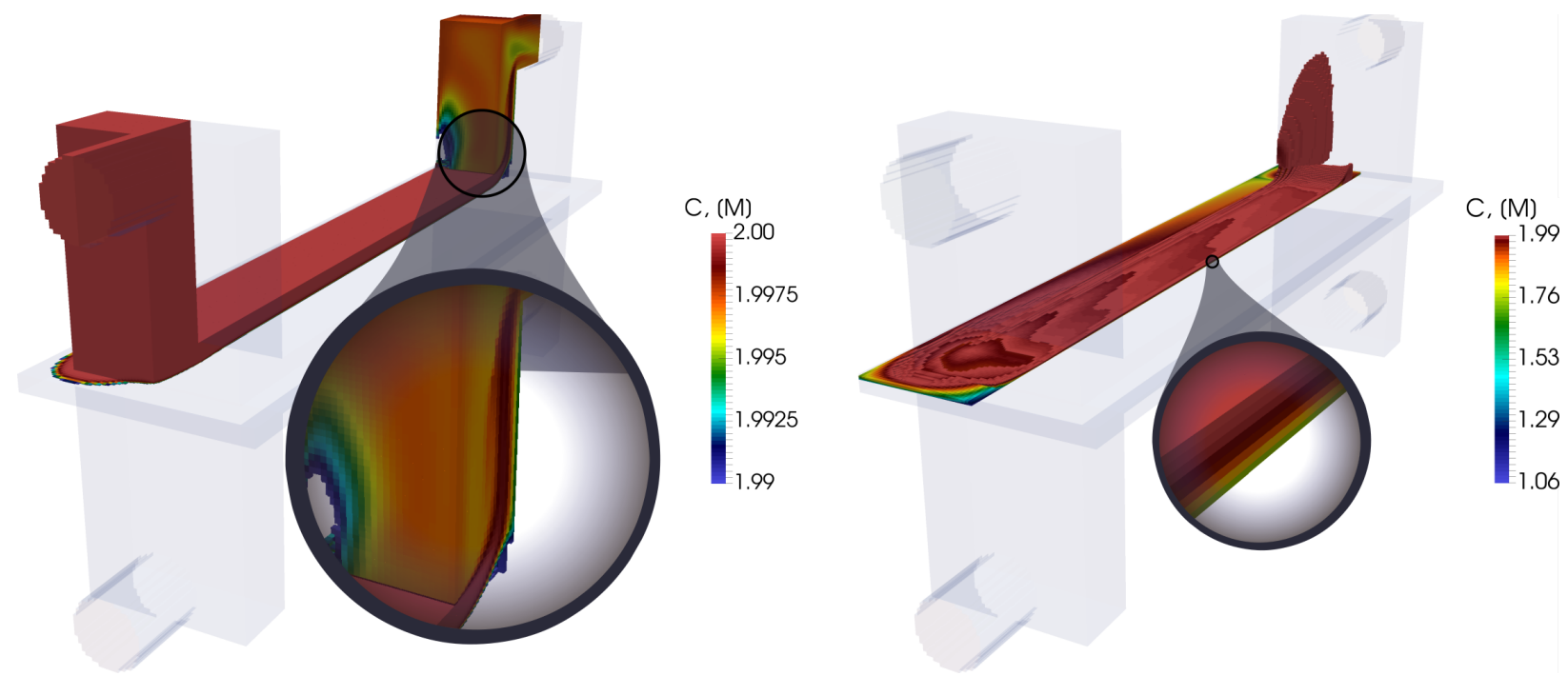

Figure 10: Concentration distribution in the draw channel. We split the draw channel into two regions: one with concentration between $1.99 M$ and $2 M$ on the left and another with concentration between $1.06 M$ and $1.99 M$ on the right.
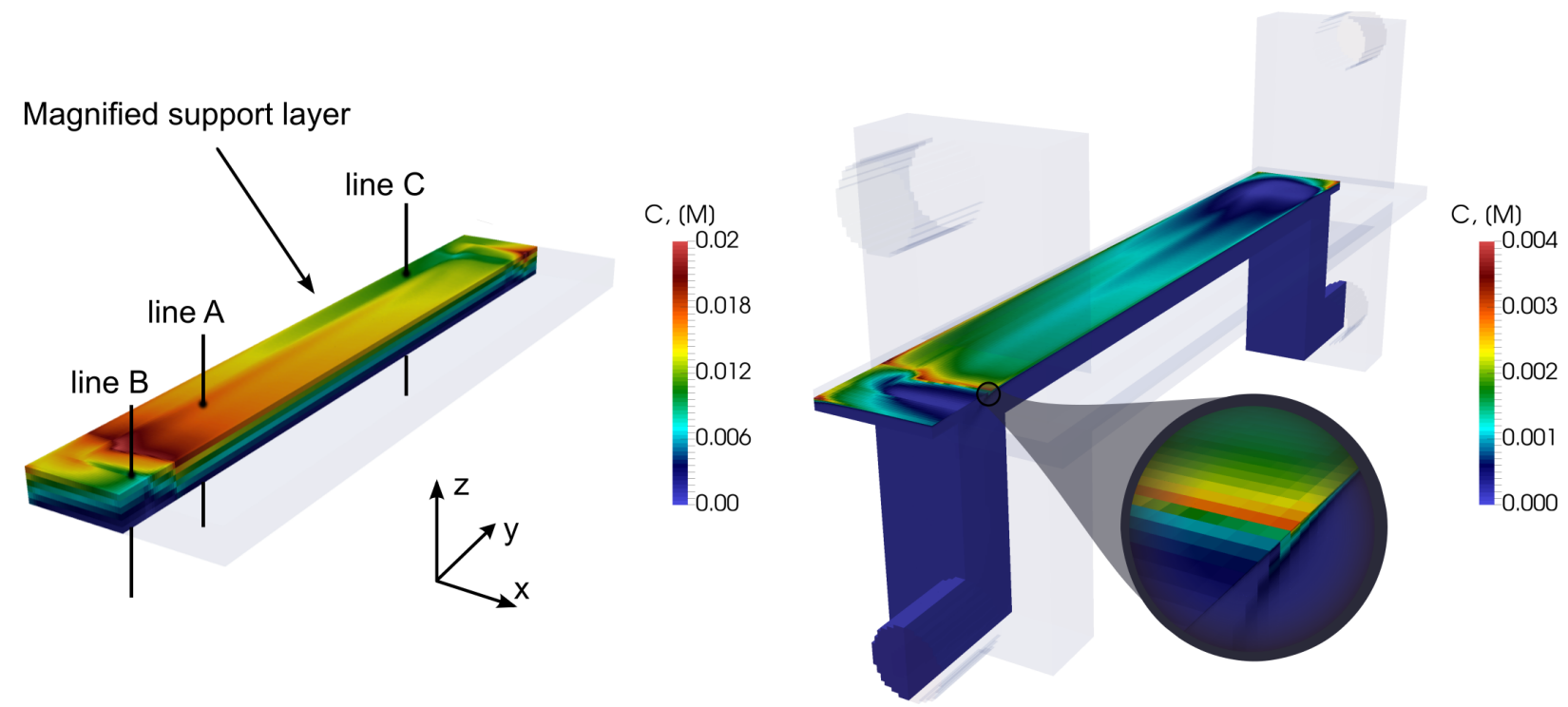

Figure 11: On the left, the concentration distribution inside of the support layer with magnified thickness is shown. On the right, the concentration distribution in the feed channel is shown. 


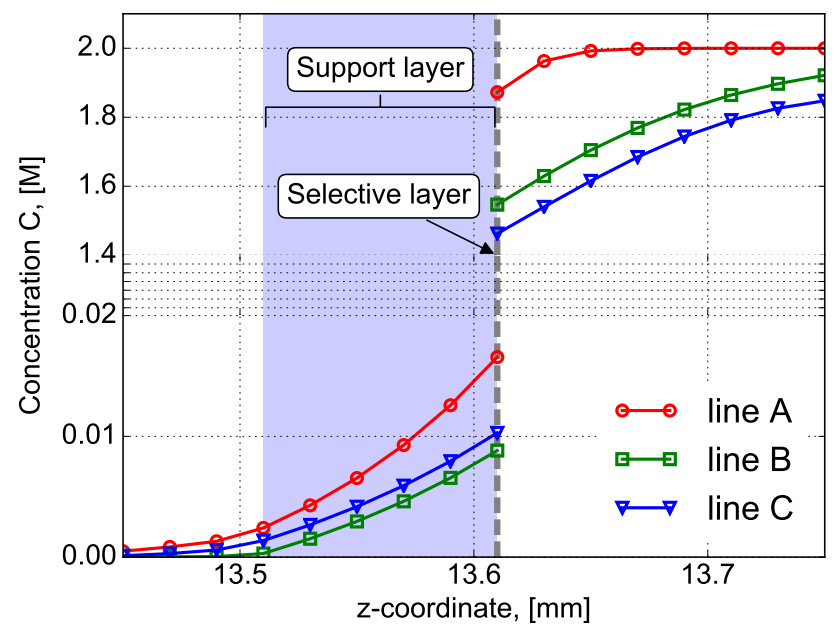

Figure 12: Concentration distribution plotted over lines A, B, and C (see Figure 11).

Figure 10 presents the distribution of the concentration in the draw channel. We use a threshold of $1.99 M$ for the concentration to split the draw channel into two regions: one with the concentration between $1.99 M$ and $2 M$ (the left of Figure 10) and another one with the concentration between $1.06 M$ and $1.99 M$ (the right of Figure 10). The domain obtained for the former case has almost constant maximum concentration. Only close to the draw outlet we see some variations in the concentration values, which occur due to the water flux through the membrane dissolving the draw solution (see magnified region in the left of Figure 10). The domain obtained for the latter case shows the region where the effect of the external concentration polarization appears (see magnified region in the right of Figure 10). The left of Figure 11 shows the distribution of the concentration inside the support layer, which also represents the internal concentration polarization. The right of Figure 11 shows concentration distribution in the feed channel, where concentration values are very small. Hence, the external concentration polarization on the side of the feed channel is negligible in AL-DS mode. Showing concentration distribution in 3D provides us with a complete picture, but makes the representation less intuitive. Figure 12 shows 1D concentration distributions at three randomly chosen locations marked as lines A, B, and C in the left of Figure 11. Here, we observe that the internal and external concentration polarizations have different shapes and magnitudes depending on the position at which the concentration distribution is considered. Thus, the concentration distribution has a 3D behavior due to the shape of the cell element and the heterogeneous fluid flow.

Figure 13 shows the water and solute fluxes through the selective layer of the membrane, where the direction of the flow in the draw channel is from the left to the right. Similarly to the velocity and concentration distributions, we observe heterogeneous distributions of the water and solute fluxes through the selective layer. Moreover, the water and solute fluxes occur in opposite directions. In the forward osmosis, diffusion dominates convection across the membrane. Therefore, while the water flux occurs in the direction from the low to high osmotic pressures, that is from the feed to draw channels, the solute flux caused by the diffusion transport mechanism occurs in the direction from the draw to feed channels.

The experimental data is analyzed assuming that the flow is fully parallel to the membrane and all distributions are homogeneous. But the simulations demonstrate the influence of the shape of the cell element on the behavior of the forward osmosis experiment.

\section{2. $2 D$ vs. $3 D$ simulations}

Performing 2D simulations is easier, since no complex 3D cell prototypes need to be constructed. Additionally, the 2D simulations are faster than the 3D ones. Therefore, the 2D simulations are usually preferred. In this section we quantitatively study the influence of the heterogeneities of the velocity and concentration distributions observed in Section 4.1 on the performance of the membrane and we determine if the reduction of the dimensionality from $3 \mathrm{D}$ to $2 \mathrm{D}$ is reasonable using our cell element for the forward osmosis experiments. 


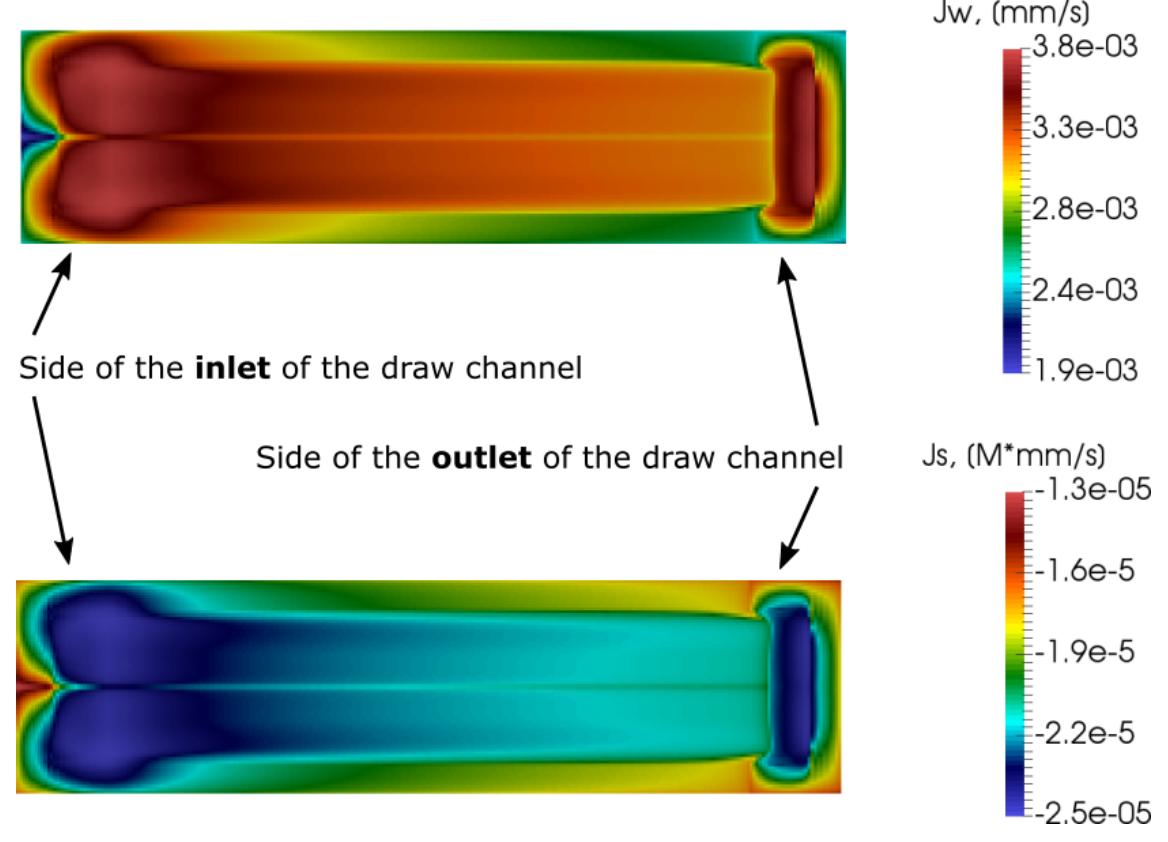

Figure 13: The water and solute fluxes through the selective layer are shown on the left and on the right, respectively.

We perform a series of 3D forward osmosis numerical experiments in AL-DS modes for the same cell element (Figure 7) varying simultaneously flow rates in the draw and feed channels which take the following values: $0.025,0.05,0.075,0.1,0.125$ and $0.15 \mathrm{~L} / \mathrm{min}$. Correspondingly, we run $2 \mathrm{D}$ forward osmosis numerical experiments in AL-DS mode

- preserving height and length of the cross-flow channels, respectively;

- using the same numerical parameters (see Table 4) and discretization (see Figure 14);

- and prescribing average cross-flow velocities for 2D case instead of flow rates. The averaged cross-flow velocities are computed using the flow rates and the area of the cross-section of the cross-flow channels in $3 \mathrm{D}$ case.

Each $2 \mathrm{D}$ simulation takes in average $38 \mathrm{~min}$.

To estimate the cost of the dimensionality reduction, we compute a relative error of the averaged water flux across the membrane obtained in the $2 \mathrm{D}$ problems in comparison with the averaged water flux in $3 \mathrm{D}$ problems. The averaged water flux across the membrane is denoted by $J_{w}$ and is computed as follows

$$
J_{w}=\frac{1}{m\left(\Gamma^{i}\right)} \int_{\Gamma^{i}} \mathbf{u} \cdot \mathbf{n} d \sigma
$$

where $m(\cdot)$ is the area of the membrane.

Figure 15 shows the dependence of the relative error on the flow rate. The simulations show that the error is a nonlinear function of the flow rate and it can be up to $12 \%$ for flow rate $0.025 \mathrm{~L} / \mathrm{min}$ and larger for smaller flow rates. Thus, our cell element designed for forward osmosis experiments introduces an error in the range of $3-12 \%$ to the membrane performance. Despite the heterogeneities observed in the 3D simulations (see Section 4.1), the quantitative analysis shows that the error between 2D and 3D simulations is small and can be neglected for the forward osmosis experiments using this cell element. 

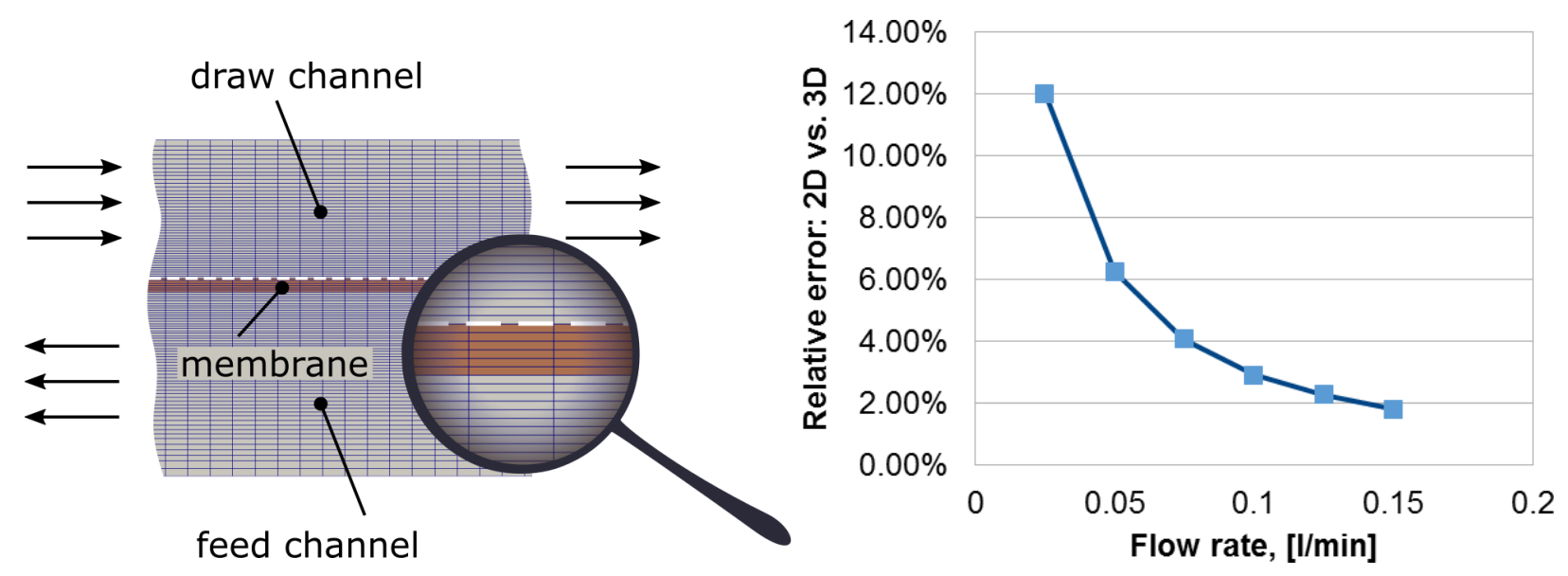

Figure 14: Irregular mesh for 2D simulations with marked flow Figure 15: Relative error of the averaged water flux across directions and cross-flow channels. the membrane obtained in $2 \mathrm{D}$ problem in comparison with the averaged water flux in $3 \mathrm{D}$ problem.

\section{Conclusions and discussions}

We present a new module of the simulation tool PoreChem for modeling osmotic processes at the cellelement scale and performing parametric studies. The mathematical model describes forward osmosis processes in an arbitrary 3D domain, which allows us to perform 3D simulations of the processes in cell elements commonly used in laboratory experiments. The mathematical model accounts for nonlinear osmotic pressureconcentration relation and, thus, can be used for different osmotic processes accounting for characteristics of the support layer. From the results detailed above we can draw the following conclusions.

- The mathematical validation shows that the flow model and algorithm have the correct behavior and produce reliable results.

- We observed that the distributions of the velocity, the concentration, and the water and solute fluxes through the selective layer are affected by the shape of the cell element in a forward osmosis experiment with the selective layer facing the draw solution.

- We examine the quality of our cell element and show that when using approximate 2D model instead of $3 \mathrm{D}$ full one in the forward osmosis simulations, with the selective layer facing the draw solution, the error in calculating the membrane performance is in the range of $3-12 \%$.

When reducing the dimensionality of simulations of forward osmosis in a cell element (using a 2D model instead of 3D one), we commit an error. For example, in our case the error is $3-12 \%$ of the membrane performance. This means that if laboratory measurements obtained with this cell element are used as an input for the 2D model to calculate intrinsic properties of the membrane, e.g., intrinsic water and solute permeability coefficients $[1,20]$ or the membrane structural parameter [17], the obtained properties may have a $3-12 \%$ error. In engineering designs where errors of order $10 \%$ are acceptable, $2 \mathrm{D}$ simulations might be preferable since they are faster to perform and easier to setup and analyze than 3D simulations. However, in order to decide if the 3D nature of the process is important or if the error committed by using a $2 \mathrm{D}$ model is significant for the case of interest, a $3 \mathrm{D}$ validation needs to be performed for a given cell geometry and range of parameters.

The software simulation tool PoreChem has some other potential applications. For example PoreChem can be used to predict membrane performance under different operational conditions as well as to improve the design and to optimize the cell elements. We could predict how a membrane will behave in laboratory scale experiments and in industrial scale. This model accounts for the heterogeneous permeability of the selective layer (see Appendix A) and of the support layer to study the effect of the membrane heterogeneities and defects. 


\section{Acknowledgements}

This publication was made possible in part by the CSIRO Professorial Chair in Computational Geoscience at Curtin University and the Deep Earth Imaging Enterprise Future Science Platforms of the Commonwealth Scientific Industrial Research Organisation, CSIRO, of Australia. Additional support was provided by the European Union's Horizon 2020 Research and Innovation Program of the Marie Skłodowska-Curie grant agreement No.644202, the Mega-grant of the Russian Federation Government (N 14.Y26.31.0013), the Curtin Institute for Computation, and by the Center for Numerical Porous Media at King Abdullah University of Science and Technology (KAUST). The J. Tinsley Oden Faculty Fellowship Research Program at the Institute for Computational Engineering and Sciences (ICES) of the University of Texas at Austin has partially supported the visits of VMC to ICES. Also we would like to acknowledge the funding received through the SABIC Postdoctoral Fellowship scheme at KAUST which partially supported this research project.

\section{A. Influence of selective layer with heterogeneities and defects}

This numerical example demonstrates how the mathematical model can be used to investigate the influence of defects and heterogeneities of the selective layer on the membrane performance. Now we focus only on the effective area of the membrane in AL-DS mode assuming that the effect of the shape of a cell element is eliminated. Performing simulations in a 3D cell element is straight forward, but interpretation of the results is more challenging due to superposition of effects coming from the shape of the cell element and the membrane heterogeneities. Thus, we have only two rectangular cross-flow channels with a membrane in between as Figure A.16 shows. The membrane has $5 \mathrm{~cm}$ length and $0.5 \mathrm{~cm}$ width, while the heights of the draw and feed channels are equal to $1.2 \mathrm{~cm}$ and $1.7 \mathrm{~cm}$, respectively. The selective layer is discretized by $1000 \times 100$ voxels with voxel size $50 \mu \mathrm{m}$. In the horizontal direction, perpendicular to the cross flow, we use periodic boundary conditions. We fix the constant permeability value of the support layer and consider three different cases for the permeability of the selective layer: constant, heterogeneous, and defective. The rest of the parameters are taken from the previous numerical example as summarized in Table 4.

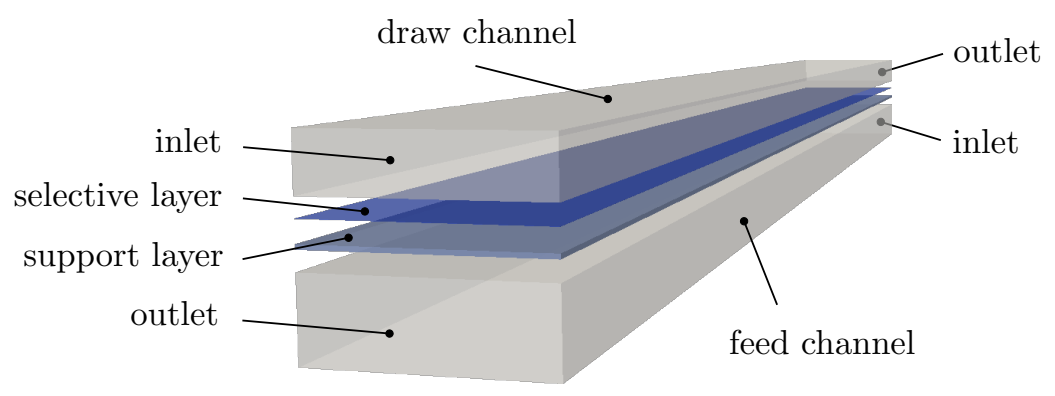

Figure A.16: Cross-flow channels for effective area of the membrane

The first numerical test is performed using constant water permeability $A=2.0 \times 10^{-17} \mathrm{~mm}^{2}$ and constant solute permeability $B=1.3 \times 10^{-5} \mathrm{~mm} / \mathrm{s}$. The second test case uses heterogeneous distributions of $A(\mathbf{x})$ and $B(\mathbf{x})$ as Figure A.17 shows, while their arithmetic averages are equal to values of the water and solute permeability from the first test case. For the third test case we use a constant permeability for the whole selective layer except 200 randomly distributed voxels, which have permeability values two orders larger (see Figure A.18). Here we consider two possibilities as shown in Figure A.18:

a) arithmetic averages of the permeability are the same as in the first test case, namely $A=2.0 \times$ $10^{-17} \mathrm{~mm}^{2}$ and $B=1.3 \times 10^{-5} \mathrm{~mm} / \mathrm{s}$

b) part of the membrane which does not have defects has permeability values the same as in the first test case, then total arithmetic averages of permeability for membrane with defects equal to $A=$ $2.4 \times 10^{-17} \mathrm{~mm}^{2}$ and $B=1.56 \times 10^{-5} \mathrm{~mm} / \mathrm{s}$. 
$\mathrm{K},\left(\mathrm{mm} \mathrm{n}^{\wedge} 2\right) \quad \mathrm{B},(\mathrm{mm} / \mathrm{s})$

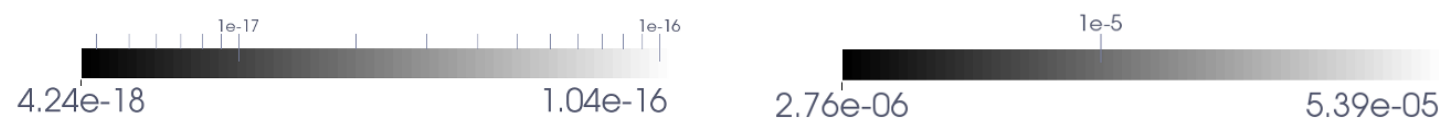

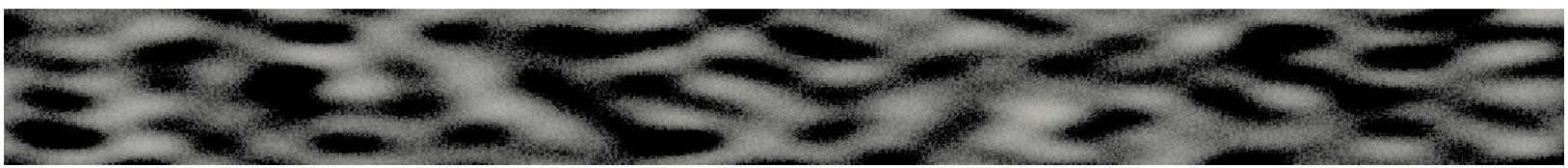

Figure A.17: Heterogeneous distributions of water and solute permeability of the selective layer

$K,(m m \wedge 2)$

a)

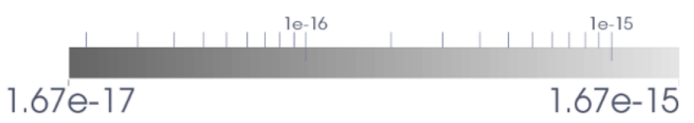

$\mathrm{K},(\mathrm{mm} \wedge 2)$

b)

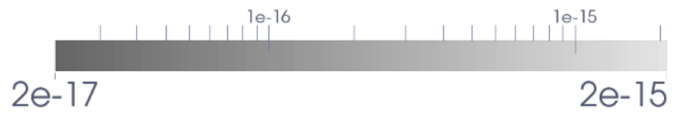

B, $(\mathrm{mm} / \mathrm{s})$

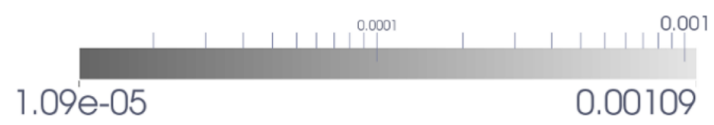

B, $(\mathrm{mm} / \mathrm{s})$

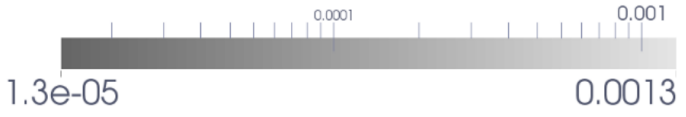

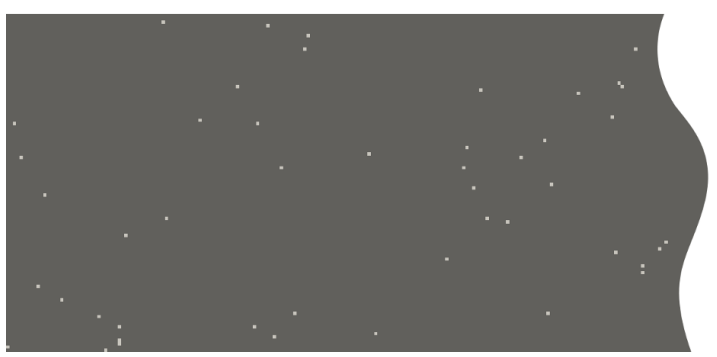

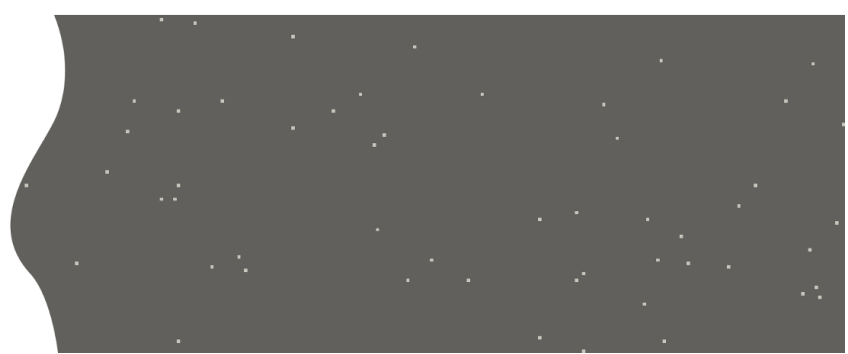

Figure A.18: Defective distributions of water and solute permeability of the selective layer

We estimate the permeability distributions as follows. For the heterogeneous case maximum and minimum permeability values defer approximately 5 times from the average value. The change in the permeability can equivalently be interpreted as the change in the thickness of the selective layer, and five-fold variations in the thickness of the selective layer seem to be possible, e.g. due to the roughness of the surface of the support layer. For the defective case the permeability change is related to the defects (breakage) of the selective layer. If a defect occurs in the selective layer, the defect is not big enough to cover the whole area of a voxel. We chose voxel size to be $50 \mu \mathrm{m}$ to be able to resolve big enough size of a membrane. Therefore, the presence of a defect in a voxel results in still porous voxel but with higher permeability. The chosen values for the heterogeneities and defects are just synthetic values and can highly vary depending on considered membrane.

In Figures A.19-A.21 we show the concentration distributions right on the top of the selective layer in the draw channel for the three test cases: constant, heterogeneous, and the defective case 'a' for permeability values $A$ and $B$. The horizontal slice lies within the ECP region and therefore the concentration distribution within this slice influences the fluxes though the membrane. Figure A.22 presents a 1D concentration distribution from the same horizontal slice as a function of the length of the membrane. The concentration is averaged over the width of the membrane. Table A.5 compares the water and solute fluxes through the selective layer for all three test cases. Although the first three tests, namely with constant (dashed blue line), 
heterogeneous (orange line), and defective case 'a' (red line) distributions of water and solute permeability $A$ and $B$, have the selective layers with the same averaged permeability values, Figure A.22 shows that they lead to different concentration distributions and as the result to different performances under the same operational conditions (see Table A.5). The fourth test on Figure A.22 and in Table A.5 shows that, as expected, a membrane with defects (green line) in comparison to a membrane with the perfect selective layer (dashed blue line) produces larger ECP and larger water flux through the membrane.

This numerical experiment demonstrates the capabilities of the general 3D mathematical model for osmotic processes and how it can be used to help investigate the other sides of the problem, such as defects and heterogeneities. Because we chose the general formulation for the model, it can be used for different regimes, also with additional pressure applied where defects have more significant impact (see discussions in [32]). However, we remark that to evaluate and examine the real impact of the heterogeneities on the membrane performance one needs to account for more accurate permeability distributions, which can be estimated from laboratory experiments using for example image techniques.

$$
\text { C, (M) }
$$

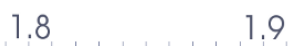

1.75 2

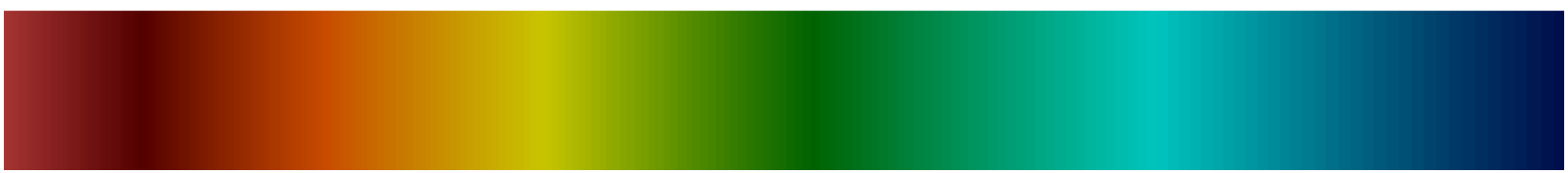

Figure A.19: External concentration polarization for the selective layer with constant permeability
1.8

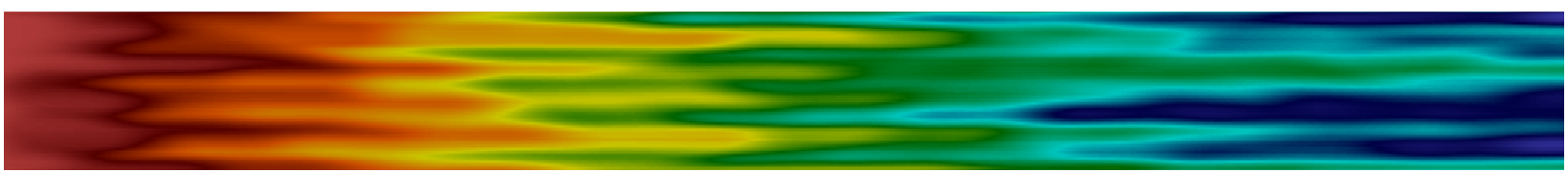

Figure A.20: External concentration polarization for the selective layer with heterogeneous permeability

\begin{tabular}{|l|c|c|c|c|}
\hline \multirow{2}{*}{$\begin{array}{l}\text { Permeability distribution type } \\
\text { of selective layer }\end{array}$} & \multicolumn{2}{|c|}{ Water flux } & \multicolumn{2}{c|}{ Solute flux } \\
\cline { 2 - 5 } & {$[L M H]$} & difference in [\%] & {$[g M H]$} & difference in [\%] \\
\hline Constant & 10.6 & - & 4.8 & - \\
\hline Heterogeneous & 10.3 & 2.8 & 4.6 & 4.2 \\
\hline Defective (case a) & 9.5 & 10.4 & 4.3 & 10.4 \\
Defective (case b) & 11.1 & 4.7 & 5 & 4.2 \\
\hline
\end{tabular}

Table A.5: Water and solute fluxes through the membrane with different types of selective layers. Columns with the title "difference in [\%]" represent differences between fluxes for the current selective layer and for the selective layer with constant permeability 


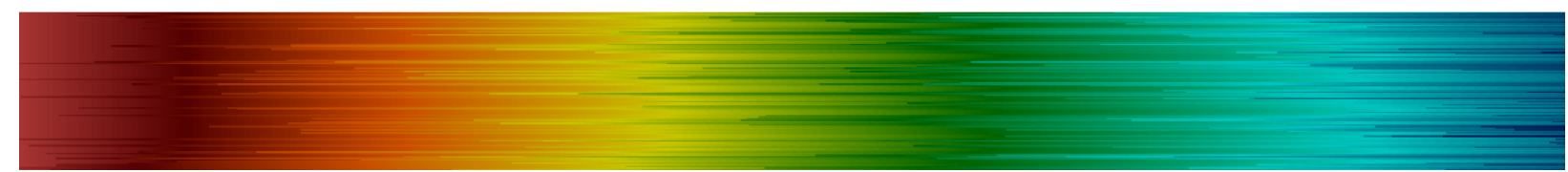

Figure A.21: External concentration polarization for the selective layer with defects $\left(A=2.0 \times 10^{-17} \mathrm{~mm}^{2}\right.$ and $B=1.3 \times$ $\left.10^{-5} \mathrm{~mm} / \mathrm{s}\right)$

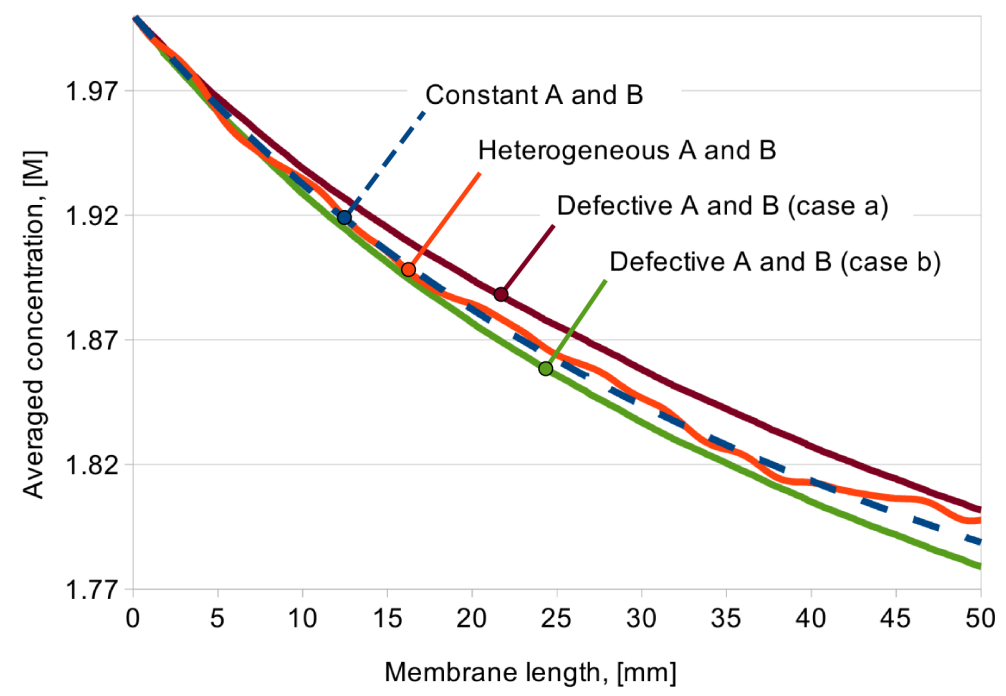

Figure A.22: Averaged external concentration polarization for all four cases

\section{References}

[1] A. Sagiv, R. Semiat, Finite element analysis of forward osmosis process using NaCl solutions, J. Membr. Sci. 379 (2011) $86-96$.

[2] J. R. McCutcheon, R. L. McGinnis, M. Elimelech, A novel ammonia-carbon dioxide forward (direct) osmosis desalination process, Desalination 174 (1) (2005) 1-11.

[3] T. Y. Cath, A. E. Childress, M. Elimelech, Forward osmosis: Principles, applications, and recent developments, J. Membr. Sci. $281(1-2)(2006) 70-87$.

[4] S. Zhao, L. Zou, C. Y. Tang, D. Mulcahy, Recent developments in forward osmosis: opportunities and challenges, J. Membr. Sci. 396 (2012) 1-21.

[5] K. Lutchmiah, A. R. D. Verliefde, K. Roest, L. C. Rietveld, E. R. Cornelissen, Forward osmosis for application in wastewater treatment: a review, Water research 58 (2014) 179-197.

[6] G. Han, S. Zhang, X. Li, T.-S. Chung, Progress in pressure retarded osmosis (PRO) membranes for osmotic power generation, Progress in Polymer Science (in press).

[7] I. L. Alsvik, M.-B. Hägg, Pressure retarded osmosis and forward osmosis membranes: Materials and methods, Polymers 5 (1) (2013) 303-327.

[8] J. R. McCutcheon, M. Elimelech, Influence of concentrative and dilutive internal concentration polarization on flux behavior in forward osmosis, J. Membr. Sci. 284 (2006) 237-247.

[9] M. Shi, G. Printsypar, O. Iliev, V. M. Calo, G. L. Amy, S. P. Nunes, Water flow prediction based on 3D membrane morphology simulation, J. Membr. Sci. 487 (2015) 19-31.

[10] C. H. Tan, H. Y. Ng, Revised external and internal concentration polarization models to improve flux prediction in forward osmosis process, Desalination 309 (2013) 125-140.

[11] G. T. Gray, J. R. McCutcheon, M. Elimelech, Internal concentration polarization in forward osmosis: role of membrane orientation, Desalination 197 (2006) 1-8. 
[12] S. Zhang, K. Wang, T. Chung, H. Chen, Y. Jean, G. Amy, Well-constructed cellulose acetate membranes for forward osmosis: Minimized internal concentration polarization with an ultra-thin selective layer, J. Membr. Sci. 375 (2011) $241-248$

[13] M. F. Gruber, C. J. Johnson, C. Y. Tang, M. H. Jensen, L. Yde, C. Hélix-Nielsen, Computational fluid dynamics simulations of flow and concentration polarization in forward osmosis membrane systems, J. Membr. Sci. 379 (2011) $488-495$.

[14] M. F. Gruber, C. J. Johnson, C. Tang, M. H. Jensen, L. Yde, C. Hélix-Nielsen, Validation and analysis of forward osmosis CFD model in complex 3D geometries, J. Membranes 2 (2012) 764-782.

[15] K. L. Lee, R. W. Baker, H. K. Lonsdale, Membranes for power generation by pressure-related osmosis, J. Membr. Sci. 8 (1981) 141-171.

[16] S. Loeb, L. Titelman, E. Korngold, J. Freiman, Effect of porous support fabric on osmosis through a Loeb-Sourirajan type asymmetric membrane, J. Membr. Sci. 129 (1997) 243-249.

[17] M. Park, J. J. Lee, S. Lee, J. H. Kim, Determination of a constant membrane structure parameter in forward osmosis processes, J. Membr. Sci. 375 (2011) 241-248.

[18] C. H. Tan, H. Y. Ng, Modified models to predict flux behavior in forward osmosis in consideration of external and internal concentration polarizations, J. Membr. Sci. 324 (2008) 209-219.

[19] C. Y. Tang, Q. She, W. C. Lay, R. Wang, A. G. Fane, Coupled effects of internal concentration polarization and fouling on flux behavior of forward osmosis membranes during humic acid filtration, J. Membr. Sci. 354 (2010) $123-133$.

[20] A. Sagiv, A. Zhu, P. D. Christofides, Y. Cohen, R. Semiat, Analysis of forward osmosis desalination via two-dimensional FEM model, J. Membr. Sci. 464 (2014) 161-172.

[21] A. P. Straub, N. Y. Yip, M. Elimelech, Raising the bar: Increased hydraulic pressure allows unprecedented high power densities in pressure-retarded osmosis, Environ. Sci. Technol. Lett. 1 (1) (2014) 55-59.

[22] A. Sagiv, P. D. Christofides, Y. Cohen, R. Semiat, On the analysis of FO mass transfer resistances via CFD analysis and film theory, J. Membr. Sci. 495 (2015) 198-205.

[23] M. Shi, G. Printsypar, P. Duong, V. Calo, O. Iliev, S. Nunes, 3D morphology design for forward osmosis, J. Membr. Sci. 516 (2016) 172-184.

[24] V. M. Calo, O. Iliev, Z. Lakdawala, K. H. L. Leonard, G. Printsypar, Pore-scale modeling and simulation of flow, transport, and adsorptive or osmotic effects in membranes: the influence of membrane microstructure, Int. J. Adv. Eng. Sci. Appl. Math. 7 (1-2) (2015) 2-13.

[25] O. Iliev, Z. Lakdawala, K. H. N. ler, T. Prill, Y. Vutov, Y. Yang, J. Yao, On the pore-scale modeling and simulation of reactive transport in 3d geometries, Mathematical Modelling and Analysis 22 (5) (2017) 671-694.

[26] M. Ehrhardt, J. Fuhrmann, A. Linke, E. Holzbecher, Mathematical modeling of channel-porous layer interfaces in PEM fuel cells, in: Proceedings of FDFC2008 - Fundamentals and Developments of Fuel Cell Conference 2008, 2008

[27] R. Ciegis, O. Iliev, Z. Lakdawala, On parallel numerical algorithms for simulating industrial filtration problems, Berichte des Fraunhofer ITWM (114).

[28] V. Laptev, Numerical solution of coupled flow in plain and porous media, Ph.D. thesis, Technical University of Kaiserslautern (2004).

[29] Z. Lakdawala, On efficient algorithms for filtration related multiscale problems, Ph.D. thesis, Technical University of Kaiserslautern (2010).

[30] K.-G. Wahlund, J. C. Griddings, Properties of an asymmetrical flow field-flow fractionation channel having one permeable wall, Anal. Chem. 59 (1987) 1332-1339.

[31] M. Park, J. Lee, C. Boo, S. Hong, S. A. Snyder, J. H. Kim, Modeling of colloidal fouling in forward osmosis membrane: Effects of reverse draw solution permeation, Desalination 314 (2013) 115-123.

[32] J. Duan, E. Litwiller, I. Pinnau, Solution-diffusion with defects model for pressure-assited forward osmosis, J. Membr. Sci. 470 (2014) 323-333. 